\title{
Exame de Papanicolaou e câncer cervical em homens transgêneros: revisão
}

\section{integrativa}

\author{
Pap smear and cervical cancer in transgender men: integrative review \\ Prueba de Papanicolaou y cáncer de cuello uterino en hombres transgéneros: revisión integradora
}

Recebido: 25/01/2021 | Revisado: 31/01/2021 | Aceito: 01/02/2021 | Publicado: 09/02/2021

\author{
Jéssica Mayara da Silva Araújo \\ ORCID: https://orcid.org/0000-0003-0453-4416 \\ Universidade Tiradentes, Brasil \\ E-mail: jessicamayara3357@gmail.com \\ Maria Mylena Gomes Santos \\ ORCID: https://orcid.org/0000-0002-9555-8175 \\ Universidade Tiradentes, Brasil \\ E-mail: myly.myh@hotmail.com \\ Renata Santos da Silva \\ ORCID: https://orcid.org/0000-0001-5459-3444 \\ Universidade Tiradentes, Brasil \\ E-mail: renatasilvasts@yahoo.com \\ Manuela de Carvalho Vieira Martins \\ ORCID: https://orcid.org/0000-0003-1222-5955 \\ Universidade Tiradentes, Brasil \\ E-mail: manuela.cvm@hotmail.com \\ Fernanda Costa Martins Gallotti \\ ORCID: https://orcid.org/0000-0002-9063-1273 \\ Universidade Tiradentes, Brasil \\ E-mail: fernanda.gallotti@souunit.com.br
}

\begin{abstract}
Resumo
Introdução: O exame de Papanicolaou é o principal método de rastreamento para o câncer do colo do útero, que pode acometer os homens transgêneros que apresentam esse órgão. Objetivo: Destacar a importância do exame citopatológico no combate ao câncer cervical em homens transgêneros. Metodologia: Trata-se de uma revisão integrativa da literatura de cunho descritivo e abordagem qualitativa, realizada nas bases SciELO, PUBMED, MEDLINE, LILACS, BVS e Science Direct, utilizando os descritores "Transgender Persons", "Uterine Cervical Neoplasms" e "Papanicolaou Test". Foram incluídos artigos de 2015 a 2020 que abordam sobre o exame de Papanicolaou, e o câncer do colo do útero ou lesões precursoras do câncer em homens transgêneros. A análise ocorreu através de leitura crítica e categorização temática. Resultados e discussão: Foram encontrados oito artigos que originaram três categorias: Rastreamento do câncer cervical em homens transgêneros, histórico relacionado ao exame de Papanicolaou e percepções sobre o rastreamento do câncer cervical. Assim, constatou-se que os homens transgêneros não realizam rastreamento do câncer cervical como as mulheres cisgêneros e enfrentam desafios únicos no exame de Papanicolaou, pertinentes a cada realidade, levando-os a buscar alternativas que minimizem seus desconfortos. Conclusão: Os dados revelam a importância do exame de Papanicolaou no rastreamento do câncer cervical em homens transgêneros, visto que comparado a meios alternativos buscados, o mesmo é o mais confiável atualmente. Ainda, reforçam a necessidade de conhecimento para um rastreamento de forma individualizada nesse público.
\end{abstract}

Palavras-chave: Pessoas transgênero; Neoplasias do colo do útero; Teste de Papanicolaou.

\begin{abstract}
Introduction: Pap smear is the main screening method for cervical cancer, which can affect transgender men who have this organ. Objective: To highlight the importance of cytopathological examination in the fight against cervical cancer in transgender men. Methodology: It is an integrative literature review of a descriptive nature and qualitative approach, carried out on the SciELO, PUBMED, MEDLINE, LILACS, VHL and Science Direct databases, using the descriptors "Transgender Persons", "Uterine Cervical Neoplasms" and "Papanicolaou Test". Articles from 2015 to 2020 that address Pap smear and cervical cancer or cancer precursor lesions in transgender men were included. The analysis took place through critical reading and thematic categorization. Results and discussion: Eight articles were found that originated three categories: Screening for cervical cancer in transgender men, history related to the Pap
\end{abstract}


Research, Society and Development, v. 10, n. 2, e17010212342, 2021

(CC BY 4.0) | ISSN 2525-3409 | DOI: http://dx.doi.org/10.33448/rsd-v10i2.12342

smear and perceptions about screening for cervical cancer. Thus, it was found that transgender men do not screen for cervical cancer like cisgender women and face unique challenges in the Pap smear, relevant to each reality, leading them to seek alternatives that minimize their discomfort. Conclusion: The data reveal the importance of the Pap smear in the screening of cervical cancer in transgender men, since compared to alternative means sought, it is the most reliable today. Still, they reinforce the need for knowledge for individualized screening in this audience.

Keywords: Transgender persons; Uterine cervical neoplasms; Papanicolaou test.

\section{Resumen}

Introducción: La prueba de Papanicolaou es el principal método de detección del cáncer de cuello uterino, que puede afectar a los hombres transgénero que tienen este órgano. Objetivo: Resaltar la importancia del examen citopatológico en la lucha contra el cáncer de cuello uterino en hombres transgénero. Metodología: Es una revisión integradora de la literatura de carácter descriptivo y abordaje cualitativo, realizada sobre las bases de datos SciELO, PUBMED, MEDLINE, LILACS, VHL y Science Direct, utilizando los descriptores "Personas Trans", "Neoplasias del Cuello Uterino" y "Prueba de Papanicolaou". Se incluyeron artículos de 2015 a 2020 que abordan el Papanicolaou y el cáncer de cuello uterino o las lesiones precursoras del cáncer en hombres transgénero. El análisis se realizó mediante lectura crítica y categorización temática. Resultados y discusión: Se encontraron ocho artículos que originaron tres categorías: Detección de cáncer de cuello uterino en hombres transgénero, antecedentes relacionados con la prueba de Papanicolaou y percepciones sobre la detección de cáncer de cuello uterino. Así, se encontró que los hombres transgénero no realizan cribado de cáncer de cuello uterino como las mujeres cisgénero y enfrentan desafíos únicos en el examen de Papanicolaou, relevantes para cada realidad, lo que los lleva a buscar alternativas que minimicen su malestar. Conclusión: Los datos revelan la importancia de la prueba de Papanicolaou en el cribado de cáncer de cuello uterino en hombres transgénero, ya que en comparación con los medios alternativos buscados, es el más confiable en la actualidad. Aún así, refuerzan la necesidad de conocimiento para una proyección individualizada en esta audiencia.

Palabras clave: Personas transgénero; Neoplasias del cuello uterino; Prueba de Papanicolaou.

\section{Introdução}

O Câncer cervical ou câncer do colo do útero tem como principal fator de predisposição a infecção persistente pelo Papilomavírus Humano (HPV), visto que por meio de microlesões pré-existentes no epitélio da ectocérvice, o vírus possui o potencial de adentrar e atingir as células basais, replicando-se através da liberação de seu DNA. A nível citológico, ocorre maturação e multiplicação demasiadamente rápida, onde as células são induzidas por oncoproteínas produzidas pelo vírus, levando ao desenvolvimento de lesões intraepiteliais, que se não tratadas podem vim a apresentar caráter neoplásico maligno (Silva, Silva, Peres, \& Oliveira, 2018).

Segundo o Instituto Nacional de Câncer, as estimativas são de 16.590 novos casos de câncer do colo do útero, sendo essa a terceira neoplasia maligna mais recorrente e a quarta causa de óbito na população feminina do Brasil. Por ser uma patologia de progressão lenta e com uma fase inicial que pode não demonstrar sintomas, é importante a detecção precoce para aumentar as chances de tratamento (Brasil, 2020).

O principal e mais difundido método de rastreamento para neoplasias cervicais é o exame citopatológico, também conhecido como teste ou exame de Papanicolaou, pois possibilita a descoberta das alterações celulares precursoras desses cânceres, sendo essas curáveis em boa parte dos casos. As diretrizes de rastreamento brasileiras recomendam a realização a cada três anos, depois da obtenção de dois exames normais consecutivos com um intervalo de um ano entre cada um, devendo ser ofertado às mulheres na faixa etária de 25 a 64 anos e que já iniciaram atividade sexual (Brasil, 2016).

Entretanto, uma parcela das pessoas que possuem colo do útero não é rastreada conforme recomendações, pois nem todas se identificam com o sexo designado no nascimento, assumindo assim uma identidade de gênero diferente. O homem transgênero é um exemplo, tendo nascido com os órgãos reprodutivos femininos, mas se identificando como homem. Em sua maioria, por não realizarem a remoção cirúrgica dos órgãos reprodutivos, permanecem susceptíveis ao desenvolvimento de cânceres nos mesmos (Harb, Pass, Soriano, Zwick, \& Gilbert, 2019).

Os homens transgêneros apresentam maiores chances de ter um exame citopatológico inadequado, além de uma menor propensão a ter um rastreamento conforme o preconizado, quando em comparação com mulheres cisgêneros (que se 
identificam com o sexo feminino que lhes foi designado ao nascer), sendo necessário que esse público seja sempre recordado da necessidade da realização desse exame (Lam \&Abramovich, 2019).

O desejo de ignorar seus órgãos reprodutivos, a ansiedade por precisar se submeter a exames da região genital, o uso de terapia androgênica que ao decorrer do tempo acaba atrofiando o canal vaginal, bem como outros fatores psicológicos e sociais, se tornam desafios para a realização do exame de Papanicolaou nos homens transgêneros. A inexistência de dados acerca da prevalência ou do rastreamento das neoplasias cervicais uterinas nessa população, se mostra também como um obstáculo (Peitzmeier, Reisner, Harigopal, \& Potter, 2014).

Portanto, nota-se a relevância de conhecer a produção científica sobre a realização do exame citopatológico em homens transgêneros e sua relação com o câncer cervical nesses indivíduos. Assim, formulou-se a seguinte pergunta norteadora: A realização do exame citopatológico se constitui como prática essencial à rotina do homem transgênero no combate ao câncer cervical? Deste modo, esta pesquisa objetiva destacar a importância do exame citopatológico no combate ao câncer cervical em homens transgêneros.

\section{Metodologia}

Trata-se de uma revisão integrativa da literatura de cunho descritivo e abordagem qualitativa. Segundo Souza, Silva e Carvalho (2010), essa se configura como a mais ampla abordagem metodológica no que se refere às revisões existentes, pois incorpora uma variedade de propósitos: definir conceitos, revisar teorias e evidências, e analisar problemas metodológicos de tópicos específicos.

Para a síntese deste estudo, seguiram-se as seguintes fases da revisão integrativa: elaboração da pergunta norteadora, busca ou amostragem na literatura, coleta de dados, análise crítica dos estudos incluídos, discussão dos resultados e apresentação da revisão (Souza, Silva, \& Carvalho, 2010).

O levantamento dos artigos foi realizado entre março e maio de 2020 nas bases Scientific Electronic Library Online (SciELO), National Library of Medicine (PUBMED), Medical Literature Analysis and Retrieval System Online (MEDLINE), Literatura Latino-Americana e do Caribe em Ciências da Saúde (LILACS), Biblioteca Virtual em Saúde (BVS) e Science Direct. Para tanto, utilizou-se os Descritores em Ciências da Saúde: “Transgender Persons”, “Uterine Cervical Neoplasms” e "Papanicolaou Test” utilizando o operador booleano AND, resultando na combinação: “Transgender Persons” AND "Uterine Cervical Neoplasms" AND "Papanicolaou Test".

Os critérios de inclusão para selecionar a amostra foram: artigos com texto completo; publicados no período de 2015 a maio de 2020; nos idiomas inglês, português e espanhol; que abordam sobre o exame de Papanicolaou, e sobre câncer cervical ou lesões precursoras do câncer em homens transgêneros. Foram excluídos os artigos em duplicata, formato de tese, dissertação, revisões de literatura, carta editorial e capítulos de livros.

Visando organizar a amostra final para posterior análise, elaborou-se um instrumento de coleta de dados de forma a destacar: título, autor (es), ano de publicação, delineamento do estudo, país, idioma, base de dados, objetivos e resultados.

Procedeu-se a leitura na íntegra de cada artigo, pelas três autoras separadamente, a fim de verificar se atendiam a todos os critérios de inclusão e exclusão estabelecidos. Quaisquer discordâncias surgidas quanto à inclusão ou exclusão foram resolvidas pelas autoras em conjunto. A amostra final foi analisada mediante leitura crítica, que possibilitou comparar e associar os artigos, propiciando a definição de categorias temáticas.

Por se tratar de uma revisão de literatura, onde os materiais a serem analisados são de domínio público, não houve necessidade de submissão a Comitê de Ética em Pesquisa. 
Research, Society and Development, v. 10, n. 2, e17010212342, 2021

(CC BY 4.0) | ISSN 2525-3409 | DOI: http://dx.doi.org/10.33448/rsd-v10i2.12342

\section{Resultados e Discussão}

Através da busca nas bases de dados, foram encontrados 35 artigos, dos quais oito constituíram a amostra final. A esquematização do levantamento dos estudos encontra-se representada na Figura 1.

Figura 1 - Fluxograma da seleção dos artigos incluídos.

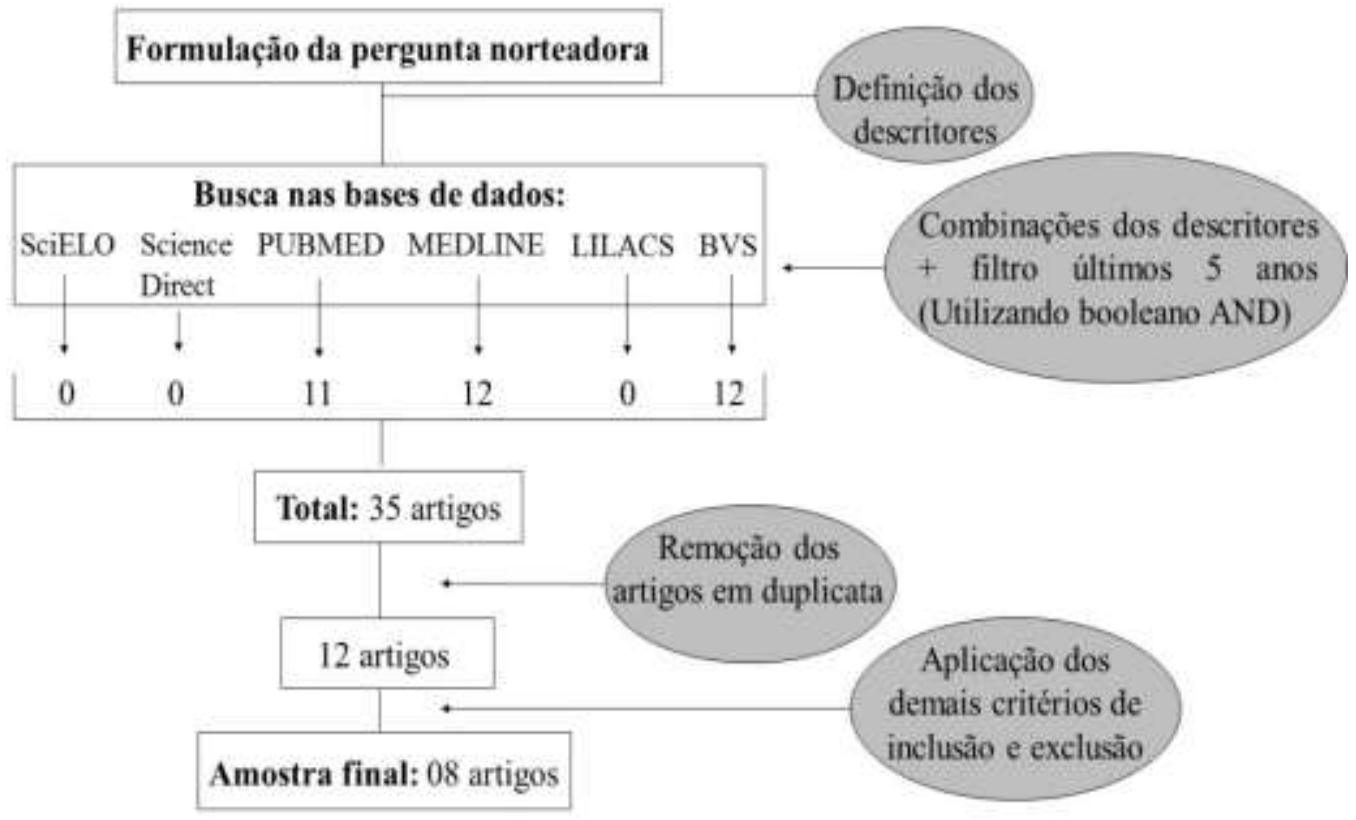

Fonte: Autores (2020).

A distribuição dos artigos nas bases de dados demonstrou que as oito publicações se encontram simultaneamente na PUBMED, MEDLINE e BVS. Nas demais bases de dados pesquisadas não houve resultados para os descritores utilizados. Dos oito artigos incluídos, sete foram desenvolvidos nos Estados Unidos e um no Canadá. Em relação ao idioma de publicação, houve predominância do inglês. Dos estudos selecionados, um foi publicado em 2019, dois em 2016 e 2018, e três em 2017, ano com maior produção científica sobre o tema pesquisado. Os principais resultados identificados nos estudos foram destacados no Quadro 1.

Quadro 1 - Caracterização da amostra quanto ao título, ano de publicação, delineamento do estudo, objetivos e resultados.

\begin{tabular}{|l|l|l|l|}
\hline Título (Ano) & $\begin{array}{l}\text { Delineamento do } \\
\text { Estudo }\end{array}$ & Objetivos & Resultados \\
\hline $\begin{array}{l}\text { Perceptions of cervical } \\
\text { cancer risk and screening } \\
\text { among transmasculine } \\
\text { individuals: patient and } \\
\text { provider perspectives (2016) }\end{array}$ & $\begin{array}{l}\text { Estudo qualitativo guiado por } \\
\text { princípios da teoria } \\
\text { fundamentada baseado em } \\
\text { entrevistas e grupos focais. }\end{array}$ & $\begin{array}{l}\text { Verificar como as percepções } \\
\text { de risco de indivíduos } \\
\text { transmasculinos e } \\
\text { profissionais de saúde podem } \\
\text { influenciar o uso do exame } \\
\text { Papanicolau entre pessoas no } \\
\text { continuo transmasculino. }\end{array}$ & $\begin{array}{l}\text { No geral, os pacientes creem que indivíduos } \\
\text { transmasculinos devem realizar exames de } \\
\text { Papanicolaou regulares. A maioria acreditava que } \\
\text { difere biologicamente devido a identidade de } \\
\text { gênero, porém profissionais acreditam que homens } \\
\text { trans possuem comportamento de risco menor que } \\
\text { as mulheres cisgênero. }\end{array}$ \\
\hline
\end{tabular}


Research, Society and Development, v. 10, n. 2, e17010212342, 2021 (CC BY 4.0) | ISSN 2525-3409 | DOI: http://dx.doi.org/10.33448/rsd-v10i2.12342

\begin{tabular}{|c|c|c|c|}
\hline $\begin{array}{l}\text { Gender identity disparities in } \\
\text { Pap test use in a sample of } \\
\text { binary and non-binary } \\
\text { transmasculine adults (2018) }\end{array}$ & $\begin{array}{l}\text { Estudo baseado em uma } \\
\text { pesquisa autoaplicada em } \\
2015-2016 \text {. }\end{array}$ & $\begin{array}{l}\text { Examinar se a triagem do } \\
\text { câncer cervical difere entre } \\
\text { nascidos mulheres } \\
\text { identificados como homens, } \\
\text { homens trans ou FTM } \\
\text { (binários) e aqueles com } \\
\text { outra identidade } \\
\text { transmasculina (não- } \\
\text { binários). }\end{array}$ & $\begin{array}{l}\text { Os indivíduos em sua maioria brancos, com ensino } \\
\text { superior e seguro de saúde, tiveram maior } \\
\text { prevalência na realização do exame de } \\
\text { Papanicolaou que as mulheres cisgênero em 2015, } \\
\text { nos Estados Unidos. Alguns indivíduos relataram } \\
\text { problemas no acesso aos serviços de saúde nos } \\
\text { últimos } 12 \text { meses e evitaram atendimento por } \\
\text { medo de discriminação. Nos últimos três anos, } \\
77,1 \% \text { dos participantes tinham feito um exame de } \\
\text { Papanicolaou. }\end{array}$ \\
\hline $\begin{array}{l}\text { Understanding Transgender } \\
\text { Men's Experiences with and } \\
\text { Preferences for Cervical } \\
\text { Cancer Screening: A Rapid } \\
\text { Assessment Survey (2017) }\end{array}$ & $\begin{array}{l}\text { Pesquisa de avaliação rápida } \\
\text { com abordagem de pesquisa } \\
\text { participativa baseada na } \\
\text { comunidade. }\end{array}$ & $\begin{array}{l}\text { Compreender experiências e } \\
\text { preferências para o } \\
\text { rastreamento do câncer } \\
\text { cervical entre homens trans. }\end{array}$ & $\begin{array}{l}\text { O equivalente a 50,5\% dos participantes não } \\
\text { realizou exame de Papanicolaou nos últimos três } \\
\text { anos. } 57,1 \% \text { relatam preferir a auto amostragem } \\
\text { do HPV como alternativa ao exame de } \\
\text { Papanicolaou coletado pelo profissional. }\end{array}$ \\
\hline $\begin{array}{l}\text { Locally advanced cervical } \\
\text { cancer in a transgender man } \\
(2019)\end{array}$ & Relato de Caso & $\begin{array}{l}\text { Relatar um caso de câncer } \\
\text { cervical localmente avançado } \\
\text { em um homem trans e a } \\
\text { realização do exame } \\
\text { Papanicolaou. }\end{array}$ & $\begin{array}{l}\text { O homem transgênero de } 45 \text { anos foi informado } \\
\text { que não precisava realizar rastreamento para o } \\
\text { câncer cervical, pois nunca havia tido relações } \\
\text { sexuais penetrantes com um homem. Após sinais e } \\
\text { sintomas, um exame de Papanicolaou confirmou } \\
\text { lesão intraepitelial escamosa de alto grau com } \\
\text { suspeita de invasão. Após biópsias confirmando } \\
\text { carcinoma cervical de células escamosas, o } \\
\text { paciente foi tratado. }\end{array}$ \\
\hline $\begin{array}{l}\text { Characteristic findings of } \\
\text { cervical Papanicolaou tests } \\
\text { from transgender patients on } \\
\text { androgen therapy: } \\
\text { Challenges in detecting } \\
\text { dysplasia (2018) }\end{array}$ & $\begin{array}{l}\text { Revisão retrospectiva de } \\
\text { relatórios de um sistema de } \\
\text { informações de patologia } \\
\text { entre } 2000 \text { e } 2017 \text {. }\end{array}$ & $\begin{array}{l}\text { Estabelecer as características } \\
\text { do teste de Papanicolaou na } \\
\text { população de transgêneros da } \\
\text { FTM (espectro de mulheres } \\
\text { para homens) em terapia com } \\
\text { andrógenos. }\end{array}$ & $\begin{array}{l}57 \% \text { dos exames de Papanicolaou foram negativos } \\
\text { para lesão intraepitelial, } 13 \% \text { insatisfatórios, } 13 \% \\
\text { com células escamosas atípicas de significado } \\
\text { indeterminado, } 13 \% \text { com células escamosas } \\
\text { atípicas - não descartando lesão intraepitelial } \\
\text { escamosa de alto grau e } 4 \% \text { de lesão intraepitelial } \\
\text { escamosa de alto grau. }\end{array}$ \\
\hline $\begin{array}{l}\text { Cervical Cancer Screening } \\
\text { Preferences Among Trans } \\
\text { Masculine Individuals: } \\
\text { Patient-Collected Human } \\
\text { Papillomavirus Vaginal } \\
\text { Swabs Versus Provider- } \\
\text { Administered Pap Tests } \\
\text { (2017) }\end{array}$ & $\begin{array}{l}\text { Estudo de métodos mistos } \\
\text { baseado em entrevistas } \\
\text { pessoais em profundidade e } \\
\text { pesquisa online. }\end{array}$ & $\begin{array}{l}\text { Elucidar as preferências de } \\
\text { rastreamento do câncer } \\
\text { cervical entre os indivíduos } \\
\text { transmasculinos. }\end{array}$ & $\begin{array}{l}\text { A maioria dos participantes preferiram a coleta } \\
\text { através de swab para o teste de HPV realizada por } \\
\text { eles mesmos ou pelo profissional, no lugar do } \\
\text { exame de Papanicolaou ( } 94 \% \text { nas entrevistas } \\
\text { pessoais e } 91 \% \text { online). A percepção foi que a } \\
\text { coleta através do swab é menos invasiva e gera } \\
\text { menos discordância de gênero que o exame. }\end{array}$ \\
\hline $\begin{array}{l}\text { Quantitative and Mixed } \\
\text { Analyses to Identify Factors } \\
\text { that Affect Cervical Cancer } \\
\text { Screening Uptake among } \\
\text { Lesbian and Bisexual } \\
\text { Women and Transgender } \\
\text { Men (2016) }\end{array}$ & $\begin{array}{l}\text { Estudo de métodos mistos } \\
\text { convergente-paralelo baseado } \\
\text { em questionário online e } \\
\text { entrevistas telefônicas } \\
\text { detalhadas. }\end{array}$ & $\begin{array}{l}\text { Medir a prevalência e } \\
\text { identificar fatores associados } \\
\text { à triagem do câncer cervical } \\
\text { em uma amostra de lésbicas, } \\
\text { bissexuais, mulheres queer e } \\
\text { homens trans. }\end{array}$ & $\begin{array}{l}73 \% \text { da amostra realizava rastreamento de rotina. } \\
\text { Nos indivíduos FTM e gênero Queer, } 12,3 \% \text { são } \\
\text { rastreadores rotineiros e } 24,1 \% \text { rastreadores não } \\
\text { rotineiros. Quando o indivíduo se identifica com o } \\
\text { gênero feminino, a probabilidade de realizar } \\
\text { exames de Papanicolaou de rotina é maior em } \\
\text { comparação aquele que se identifica com outro } \\
\text { gênero. }\end{array}$ \\
\hline
\end{tabular}


Research, Society and Development, v. 10, n. 2, e17010212342, 2021

(CC BY 4.0) | ISSN 2525-3409 | DOI: http://dx.doi.org/10.33448/rsd-v10i2.12342

\begin{tabular}{|c|c|c|c|}
\hline $\begin{array}{l}\text { "It Can Promote an } \\
\text { Existential Crisis": Factors } \\
\text { Influencing Pap Test } \\
\text { Acceptability and Utilization } \\
\text { Among Transmasculine } \\
\text { Individuals (2017) }\end{array}$ & $\begin{array}{l}\text { Estudo qualitativo com } \\
\text { abordagem modificada da } \\
\text { teoria fundamentada baseado } \\
\text { em entrevistas } \\
\text { semiestruturadas em } \\
\text { profundidade. }\end{array}$ & $\begin{array}{l}\text { Informar intervenções } \\
\text { baseadas em evidências para } \\
\text { promover a triagem regular } \\
\text { do câncer cervical na } \\
\text { população transmasculina. }\end{array}$ & $\begin{array}{l}\text { Os indivíduos que conseguiam reformular o exame } \\
\text { de Papanicolaou como masculino ou neutro, } \\
\text { relatavam um estresse emocional mínimo. Já os } \\
\text { que não conseguiam o tinham como } \\
\text { emocionalmente difícil. Participantes afirmam que } \\
\text { profissionais trans-competentes poderiam reduzir } \\
\text { as barreiras à triagem. }\end{array}$ \\
\hline
\end{tabular}

Fonte: Autores (2020).

A análise dos artigos associada ao objetivo e à pergunta norteadora deste estudo indicou três categorias temáticas, sendo elas:

\section{Rastreamento do câncer cervical em homens transgêneros}

Alguns estudos demonstram que os indivíduos transgêneros não estão realizando o rastreamento do câncer cervical na mesma proporção que as mulheres cisgêneros (Johnson, Mueller, Eliason, Stuart, \& Nemeth, 2016; Seay et al., 2017), e as probabilidades de realização rotineira do exame de Papanicolaou variam a depender da identidade ou expressão de gênero assumida, onde os que se reconhecem como androgénos, transgêneros ou gênero queer apresentam menores chances, em comparação a expressões de gênero femininas (Johnson et al., 2016), e ainda, observa-se que essas chances também são menores nos indivíduos transgêneros binários quando comparados aos não binários (Agénor et al., 2018).

Ao verificar as taxas de rastreamento do câncer cervical em pessoas transgêneros, apenas um estudo encontrou índices mais elevados em comparação com as mulheres cisgêneros, demonstrando uma maior predominância na execução do exame na população trans (Agénor et al., 2018), porém, um aspecto a ser considerado nesse estudo são as características dos indivíduos analisados, que em sua maioria eram brancos, segurados, e possuíam formação superior. Essas informações sugerem que aqueles que possuem melhores condições financeiras e sociais apresentam maior adesão ao rastreamento, o que não condiz com a realidade vivenciada pela totalidade.

Nota-se que a ausência de estudos desse tipo no Brasil fragiliza a análise do rastreamento na população transgênero do país, que enfrenta uma realidade caracterizada por abandono precoce dos estudos e dificuldades de adentrar no mercado de trabalho (Monteiro, Brigeiro, \& Barbosa, 2019). Os sistemas de informações em saúde disponíveis também se mostram fragilizados, pois não fornecem identificação de gênero nos dados referentes à realização do exame de Papanicolaou, podendo esconder altas taxas de pessoas trans não rastreadas.

A realização do exame de Papanicolaou no período dos últimos três anos foi considerada como rastreamento rotineiro nos métodos de alguns dos estudos analisados (Agénor et al., 2018; Johnson et al., 2016; Seay et al., 2017), e assemelha-se ao que é preconizado pelas atuais diretrizes brasileiras de rastreamento do câncer do colo do útero estabelecidas pelo Ministério da Saúde (Brasil, 2016).

A existência de alta incidência de exames de Papanicolaou anormais nos homens transgêneros quando comparadas as mulheres cisgêneros de mesma faixa etária, principalmente quando está envolvida a utilização de terapia com andrógenos (Adkins et al., 2018), requer uma maior atenção por parte dos profissionais de saúde ao prestar assistência a esse público, visto que existe uma ausência de pesquisas clínicas associando o uso da testosterona ao risco de desenvolvimento de câncer cervical (Peitzmeier et al., 2017) e diretrizes especificas para triagem nesses indivíduos.

Os desafios enfrentados pelos transgêneros no processo de rastreamento do câncer cervical envolvem em sua maioria, discriminação nos serviços de saúde, desconfortos emocionais, físicos e de gênero, e ausência de seguro de saúde 
(Seay et al., 2017; Mcdowell et al., 2017; Agénor et al., 2018; Johnson et al., 2016; Peitzmeier et al., 2017). O déficit de conhecimento adequado e experiências dos profissionais de saúde na prestação de serviços a esses indivíduos também se mostra um obstáculo (Peitzmeier et al., 2017), que necessita ser ultrapassado no intuito de proporcionar um atendimento individualizado e uma assistência assertiva a essa população.

Além disso, um estudo demonstra que indivíduos transgêneros e profissionais da saúde apresentam pouco conhecimento relacionado ao risco de infecção por HPV e câncer cervical (Agénor et al., 2016). Isso se mostra prejudicial ao processo saúde-doença dos homens transgêneros, pois afeta diretamente a procura pelos serviços de saúde, situação essa que se encontra relatada na literatura (Beswick, Corkum, \& D’Souza, 2019), resultando em falta de rastreamento e identificação tardia de neoplasias.

\section{Histórico relacionado ao exame de Papanicolaou}

Experiências de transfobia, falta de plano de saúde e o incômodo por parte do profissional e do cliente relacionado à discordância entre órgãos reprodutivos e a identidade de gênero, têm sido apontados como obstáculos para a realização de exames de Papanicolaou de maneira regular (Agénor et al., 2018). Assim como discriminações relacionadas ao gênero, orientação sexual, escolaridade, estatura e condições socioeconômicas são mais mencionadas por aqueles que não seguem o que é preconizado pelas diretrizes de rastreamento do câncer do colo do útero (Johnson et al., 2016).

A vivência do exame de Papanicolaou de forma rotineira no processo de rastreamento apresenta o potencial de promover sensação de acolhimento nos indivíduos transgêneros juntamente com seus companheiros durante a assistência de saúde, além de demonstrar uma alta probabilidade desses indivíduos referirem experiências positivas com o exame. Uma característica altamente significativa associada a adesão ao rastreamento de rotina é a recomendação do exame de Papanicolaou pelo profissional médico (Johnson et al., 2016).

Segundo o estudo de Peitzmeier e colaboradores (2017), a reformulação do exame de Papanicolaou como uma prática neutra ao gênero reduz o antagonismo entre o mesmo e a identidade de gênero dos indivíduos, tornando o exame emocionalmente menos estressor. Porém, no mesmo estudo é relatado que em se tratando de planos de saúde, assumir legalmente o gênero masculino pode levar a negação da cobertura dos exames de Papanicolaou, o que resulta em aumento da estigmatização de que o procedimento é feminino, impedindo essa reformulação e culminando na ausência de adesão.

O desconforto físico durante o exame de Papanicolaou é referido como um fator negativo frequente, suportável para a maioria dos indivíduos, porém, em alguns casos, apresenta-se como uma experiência extremamente angustiante, principalmente quando os profissionais não adaptam o exame às singularidades inerentes aos transgêneros de forma a torná-lo mais cômodo. O apoio e o respeito por parte dos profissionais, elucidando e/ou adaptando o exame de Papanicolaou para promover boas experiências, aumentam as chances dos transgêneros reorganizarem o exame como uma ação de autocuidado, influenciando na realização do exame novamente no futuro (Peitzmeier et al., 2017).

Métodos alternativos ao exame de Papanicolaou, a exemplo dos swabs vaginais, estão sendo realizados como formas de detectar precocemente a infecção causada por HPV (Agénor et al., 2016), porém quando se considera possuir seguro de saúde e ter antecedentes de triagem cervical, as chances de escolhê-los são menores. Em contrapartida, experiências passadas de discriminação e ausência de procura de serviços preventivos devido à despesa se mostram como fatores que predispõem significativamente a optar pela auto-amostragem através de swab. A existência de um histórico de experiências negativas de discriminação nos serviços de saúde associada a essas escolhas, pode espelhar a marginalização vivida pela população transgênero na sociedade (Seay et al., 2017). 
A experiência de incômodo emocional, discordância de gênero e desconforto físico através da introdução do espéculo, coleta de material cervical e estímulos sensoriais no momento da realização do exame, são considerados pelos homens transgêneros os principais fatores negativos a leva-los a optar pelo auto-swab de HPV, visto que essa técnica é considerada menos invasiva comparada ao exame de Papanicolaou, e que permite ser realizada por eles mesmos ou seus parceiros, evitando re-traumatização (Mcdowell et al., 2017).

É de extrema importância a realização de estudos que avaliem a eficácia dos auto-swabs de HPV como método de rastreamento do câncer cervical, visto que preocupações com a precisão desses exames são expressadas pelos transgêneros (Mcdowell et al., 2017). A ausência de evidências sobre a eficácia do auto-swab de HPV e outros métodos como formas de rastreamento, torna o exame de Papanicolaou a ferramenta mais confiável atualmente para o rastreamento e diagnóstico do câncer cervical.

\section{Percepções sobre o rastreamento do câncer cervical}

No que concerne ao rastreamento do câncer cervical, verifica-se que os indivíduos trangêneros, no geral, além de deterem pouco conhecimento sobre saúde ginecológica, não percebem diferenças no risco de infecção por HPV ou mesmo de desenvolver neoplasia cervical em virtude de sua identidade de gênero. Apesar de profissionais de saúde não perceberem maiores ou menores riscos biológicos para esses indivíduos, muitos atribuem as diferenças presumidas no comportamento sexual, a exemplo da redução das chances de envolvimento em práticas sexuais pênis-vaginais, a um menor risco comportamental, influenciando na necessidade do exame citopatológico (Agénor et al., 2016).

Em contrapartida, profissionais reconhecem que a transmissibilidade do HPV se associa a diversos comportamentos sexuais, além das relações pênis-vagina. Por isso, alguns consideram o histórico de práticas sexuais do indivíduo e os planos de realizar histerectomia ou terapia com testosterona para orientar quanto a realização do exame. Esse é um ponto onde há discordância entre profissionais, pois outros acreditam que recomendar a triagem baseando-se nesse histórico, pode levar indivíduos que possuem comportamento de menor risco para o HPV a evitar as diretrizes recomendadas (Agénor et al., 2016).

É possível notar divergências existentes nas percepções de alguns indivíduos transgêneros, pois enquanto uns associam a redução da triagem do câncer cervical, o uso da testosterona, as práticas sexuais peniano-vaginais com homens cisgêneros, o sexo oral, as múltiplas parcerias sexuais e a utilização de artifícios penetrantes a uma necessidade de rastreamento mais frequente, outros atribuem a ausência de anormalidades ou sinais e sintomas à falta de necessidade de rastreamento de rotina (Agénor et al., 2016), desconsiderando todos esses outros fatores e o fato de que o câncer cervical pode não demonstrar sintomas no início.

Com relação aos exames de HPV através de swab frontal, a percepção dos indivíduos transgêneros é de que se tratam de técnicas menos invasivas, que causam menos desconforto, ocasionam menor disforia de gênero e proporcionam maior sensação de controle, no caso do auto-swab, quando comparados ao exame de Papanicolaou. No entanto, alguns indivíduos expressam inquietações quanto a exatidão do auto-swab, o incômodo com a possibilidade de envolvimento com os órgãos reprodutivos e a ausência de avaliação visual pelo profissional (Mcdowell et al., 2017).

Do mesmo modo, existe preocupação com a inexistência de pesquisas clínicas associando o uso da testosterona ao risco de câncer cervical, o que faz com que os homens transgêneros acreditem que devem realizar exames de rastreamento (Peitzmeier et al., 2017), opinião corroborada por participantes de outro estudo (Seay et al., 2017). Porém, alguns indivíduos transgêneros sentem que determinados profissionais não consideram os efeitos desse hormônio na elasticidade e na atrofia do canal vaginal, procedendo ao exame de Papanicolaou sem esforçar-se para proporcionar conforto (Peitzmeier et al., 2017). 
Research, Society and Development, v. 10, n. 2, e17010212342, 2021

(CC BY 4.0) | ISSN 2525-3409 | DOI: http://dx.doi.org/10.33448/rsd-v10i2.12342

Além disso, existe uma associação por parte de muitos indivíduos transgêneros, entre as identidades de gênero mais fortemente masculinas e uma disforia extrema ao realizar um exame de Papanicolaou, enquanto os gêneros queer ou "fluido" se associam a maior tranquilidade. Muitas das vezes, as percepções dos pacientes sobre o exame são modeladas negativamente, tanto pelo uso de terminologias femininas, quanto pela organização do ambiente com pôsteres apresentando mulheres, interferindo na concepção do exame e fazendo-o ser considerado um procedimento feminino (Peitzmeier et al., 2017).

A produção cientifica atual sobre a realização do exame de Papanicolaou e o câncer cervical nos homens transgêneros apresenta-se escassa. Apenas oito artigos discorrem sobre essa temática, e os objetivos e delineamentos metodológicos não se encontram devidamente esclarecidos em todos eles, necessitando de uma inferência por parte das autoras desta revisão. Em sua maioria, as amostras selecionadas nas pesquisas eram relativamente pequenas, e por isso, os dados encontrados podem não refletir a realidade dos indivíduos transgêneros em geral.

\section{Conclusão}

Decorrente da revisão, conclui-se que os homens transgêneros não realizam o rastreamento do câncer cervical do mesmo modo que as mulheres cisgêneros. Dentre os desafios enfrentados pelos mesmos na realização do exame citopatológico, destacam-se a discriminação, falta de seguro de saúde, desconfortos físicos e emocionais e baixa escolaridade. O déficit de conhecimento de profissionais de saúde e de homens transgêneros sobre os riscos de infecção por HPV e câncer cervical, se mostram como obstáculos a serem superados para favorecer melhorias no rastreamento dessa neoplasia.

Apesar de ser encontrado na literatura atual métodos alternativos ao exame de Papanicolaou, ainda são necessários estudos clínicos que comprovem sua viabilidade no rastreamento do câncer cervical, o que torna o exame citopatológico, quando bem executado, a opção mais confiável atualmente no rastreio desse câncer e de suas lesões precursoras.

Devido à escassez de publicações sobre essa temática, sugere-se que pesquisas futuras busquem analisar em amostras maiores os aspectos estudados nos artigos revisados, além de verificar se existem outros fatores que podem interferir na adesão desses indivíduos ao exame. Ainda, sugere-se que os sistemas de informação em saúde brasileiros incluam categorias relacionadas ao gênero, de modo a contribuir para a condução de pesquisas e fornecer subsídios para a adoção de estratégias de rastreamento do câncer cervical nessa população.

\section{Referências}

Adkins, B. D., Barlow, A. B., Jack, A., Schultenover, S. J., Desouki, M. M., Coogan, A. C., \& Weiss, V. L. (2018). Characteristic findings of cervical Papanicolaou tests from transgender patients on androgen therapy: challenges in detecting dysplasia. Cytopathology, 29(3), $281-287$.

Agénor, M., Hughto, J. M., Peitzmeier, S. M., Potter, J., Deutsch, M. B., Pardee, D. J., \& Reisner, S. L. (2018). Gender identity disparities in Pap test use in a sample of binary and non-binary transmasculine adults. Journal of General Internal Medicine, 33(7), 1015-1017.

Agénor, M., Peitzmeier, S. M., Bernstein, I. M., McDowell, M., Alizaga, N. M., Reisner, S. L., Pardee, D. J., \& Potter, J. (2016). Perceptions of cervical cancer risk and screening among transmasculine individuals: Patient and provider perspectives. Culture, Health \& Sexuality, $18(10), 1192-1206$.

Beswick, A.; Corkum, M.; D’Souza, D. (2019). Locally advanced cervical cancer in a transgender man. Canadian Medical Association Journal, 191(3), E76E78.

Harb, C. Y. W., Pass, L. E., Soriano, I. C., Zwick, A., \& Gilbert, P. A. (2019). Motivators and Barriers to Accessing Sexual Health Care Services for Transgender/Genderqueer Individuals Assigned Female Sex at Birth. Transgender Health, 4(1), 58-67.

Instituto Nacional de Câncer. (2020). Tipos de câncer: câncer do colo do útero. https://www.inca.gov.br/tipos-de-cancer/cancer-do-colo-do-utero

Instituto Nacional de Câncer José Alencar Gomes da Silva. (2016). Diretrizes brasileiras para o rastreamento do câncer de colo do útero/Instituto Nacional de Câncer José Alencar Gomes da Silva, Coordenação de Prevenção e Vigilância, Divisão de Detecção Precoce e Apoio à Organização de Rede. Rio de Janeiro: INCA, 32-34. https://www.inca.gov.br/sites/ufu.sti.inca.local/files//media/doc ument//diretrizesparaorastreamentodocancerdocolodoutero_2016_corrigido.pdf

Johnson, M. J., Mueller, M., Eliason, M. J., Stuart, G., \& Nemeth, L. S. (2016). Quantitative and mixed analyses to identify factors that affect cervical cancer screening uptake among lesbian and bisexual women and transgender men. Journal of Clinical Nursing, 25(23-24), 3628-3642. 
Research, Society and Development, v. 10, n. 2, e17010212342, 2021 (CC BY 4.0) | ISSN 2525-3409 | DOI: http://dx.doi.org/10.33448/rsd-v10i2.12342

Lam, J. S. H., \& Abramovich, A. (2019). Transgender-inclusive care. Canadian Medical Association Journal, $191(3)$, E79.

Mcdowell, M., Pardee, D. J., Peitzmeier, S., Reisner, S. L., Agénor, M., Alizaga, N., Bernstein, I., \& Potter, J. (2017). Cervical cancer screening preferences among trans-masculine individuals: patient-collected human papillomavirus vaginal swabs versus provider-administered pap tests. LGBT Health, 4(4), 252259.

Monteiro, S., Brigeiro, M., \& Barbosa, R. M. (2019). Saúde e direitos da população trans. Cadernos de Saúde Pública, 35(4), 1-4.

Peitzmeier, S. M., Agénor, M., Bernstein, I. M., McDowell, M., Alizaga, N. M., Reisner, S. L., Pardee, D. J., \& Potter, J. (2017). “It can promote an existential crisis": factors influencing Pap test acceptability and utilization among transmasculine individuals. Qualitative Health Research, 27(14), 2138-2149.

Peitzmeier, S. M., Reisner, S. L., Harigopal, P., \& Potter, J. (2014). Female-to-male patients have high prevalence of unsatisfactory Paps compared to nontransgender females: implications for cervical cancer screening. Journal of General Internal Medicine, 29(5), $778-784$.

Seay, J., Ranck, A., Weiss, R., Salgado, C., Fein, L., \& Kobetz, E. (2017). Understanding transgender men's experiences with and preferences for cervical cancer screening: a rapid assessment survey. LGBT Health, 4(4), 304-309.

Silva, R. C. G., Silva, A. C. O., Peres, A. L., \& Oliveira, S. R. (2018). Perfil de mulheres com câncer de colo do útero atendidas para tratamento em centro de oncologia. Revista Brasileira de Saúde Materno Infantil, 18(4), 703-710.

Souza, M. T., Silva, M. D., \& Carvalho, R. (2010). Revisão integrativa: o que é e como fazer. Einstein, 8(1), $102-106$. 